\title{
Verbal retention after shadowing and after listening
}

\author{
PETER W. CAREY* \\ Lexington School for the Deaf and \\ Graduate Center, City University of New York, New York, New York 10036
}

Ss shadowed or listened to stories that had been recorded at 1 word/sec (wps), 2 wps, and 3 wps. They then took tests of word recognition, semantic retention, and syntax recognition. At the slowest rate, shadowers' word recognition and semantic retention were somewhat higher than listeners' scores, but this difference disappeared at faster rates. Significant positive correlations among all three retention scores were observed for listeners, but for shadowers word recognition was unrelated to either of the other two retention measures. The results are discussed in terms of monitoring during shadowing. Implications for experiments on selective attention are considered.

An $S$ is said to be shadowing an auditory message when he repeats it aloud word for word as he hears it. One of the first psychologists to employ the shadowing response was Cherry (1953), who asked an $S$ to shadow a message reaching one ear when a different message reached the $S$ 's other ear. Cherry employed the shadowing response not because its psycholinguistic effects were understood, but because in studying the nature of attention, he wished to have some objective evidence that an $S$ was attending to the appropriate message. $\mathrm{He}$ found that when an $\mathrm{S}$ shadowed a message on one ear, the latter was often oblivious to the message on the other ear, even to the extent of not noticing a change from English to German or from normal to backwards speech. It is conceivable that if an $\mathrm{E}$ were willing to dispense with the shadowing response, and instructed a trustworthy $S$ simply to listen to one of the messages, similar selective effects could be observed. If selective listening is possible, then it becomes important to compare the results of such an experiment with results from a shadowing experiment in order to determine if the use of shadowing introduces changes in selectivity.

Treisman has considered the question of selectivity and has written that she tried "comparing recall of the attended message when the $S$ shadowed and when he just listened and found no significant difference. But Ss do find shadowing helps them ignore the competing unattended message [personal communication,

* The work reported here was carried out at Rockefeller University and formed part of the author's PhD dissertation at Harvard University. The investigation was supported by ARPA Contract SD-187 with Harvard University to support the research of G. A. Miller. The writer gratefully acknowledges the help of G. A. Millet, D. Aaronson, T. G. Bever, A. Treisman, and D. Cross.
1966]." Her suggestion of no difference in recall after shadowing and after listening formed the impetus for the experiment reported here. The results of the experiment will be interpreted with reference to her observation that shadowing helps Ss ignore a competing unattended message. This study may be considered a first step in the exploration of the psycholinguistic and attentional effects of the shadowing response itself.

A thorough investigation, in which Ss shadowed material recorded at more than one rate and took tests on more than a single measure of retention, might reveal differences in retention between listening and shadowing. The following shadowing facilitation hypothesis was tested: Insofar as shadowing is successful, and the shadowing response that an $\mathrm{S}$ monitors is identical to the input, shadowing will have a facilitating effect on retention. When shadowing proves difficult and the response an $S$ monitors contains errors and omissions,' it will impair retention relative to listening.

\section{SUBJECTS}

The $72 \mathrm{Ss}$ in the study were students or nurses between the ages of 18 and 32 who resided in the New York City area. All were right-handed, with no known hearing or speaking disorders, and were fluent speakers of American English. They were paid for their services.

\section{STIMUIIIS MATERIALS}

Six passages of English literary prose were recorded monaurally in the E's normal intonation. A Sony Model 230 tape recorder, running at $7 \frac{1}{2} \mathrm{ips}$, was used for the master recording. Each passage contained between 250 and 263 words and was recorded at three rates: 1 word/sec (wps), 2 wps, and 3 wps. Three practice stories of similar content and length were also recorded. The first practice story was recorded at $1 \mathrm{wps}$, the second at $2 \mathrm{wps}$, and the third at 3 wps.

\section{RETENTION TESTS}

For each of the six experimental stories, three tests of retention were constructed. (1) Each word-recognition test contained three nouns, three verbs, and three adjectives selected from the story. In addition, each test contained synonyms for three other nouns, three other verbs, and three other adjectives taken from each story. The 18 words were arranged in a vertical column in random order. (2) Each semantic content test contained 14 multiple-choice questions on the informational content of the story. There were four choices for each question. The order of the questions did not correspond to the order in which the relevant information occurred in the story. (3) Each syntax-recognition test 'contained 13 multiple-choice items in random order; each item consisted of a phrase or sentence taken verbatim from the story plus two paraphrases of that phrase or sentence. The paraphrases preserved the meaning of the original phrase.

\section{DESIGN}

Every $S$ received all six stories once and all 18 tests once. One story at each of the three rates was delivered to his right ear, with no sound in his left ear, and one story at each of the three rates was delivered to his left ear, with no sound to his right ear. Across all Ss, the order in which the six stories occurred was balanced, and the order in which the right and left ears were used was also balanced. The order in which the three rates occurred was balanced across Ss for the first four stories.

\section{PROCEDURE}

There were 36 listeners and 36 shadowers. The listeners heard the three practice stories binaurally and then the six experimental stories monaurally. The three tests on a story were administered as soon as an $S$ had heard that story. All materials were played at $7 \frac{1}{2}$ ips on a Sony Model 230 tape recorder at the same average sound-pressure level of $85.5 \mathrm{~dB}$ above 0.0002 dyne $/ \mathrm{cm}^{2}$. After hearing each experimental story, an $S$ received the word-recognition test immediately. When he finished the word-recognition test, he received the content test and, on its completion, the syntax-recognition test. Ss were told that they were participating in an experiment on listening and understanding and that they were to listen carefully to the stories in order to answer the written tests on the stories. They were given no knowledge of their level of performance on the tests. 


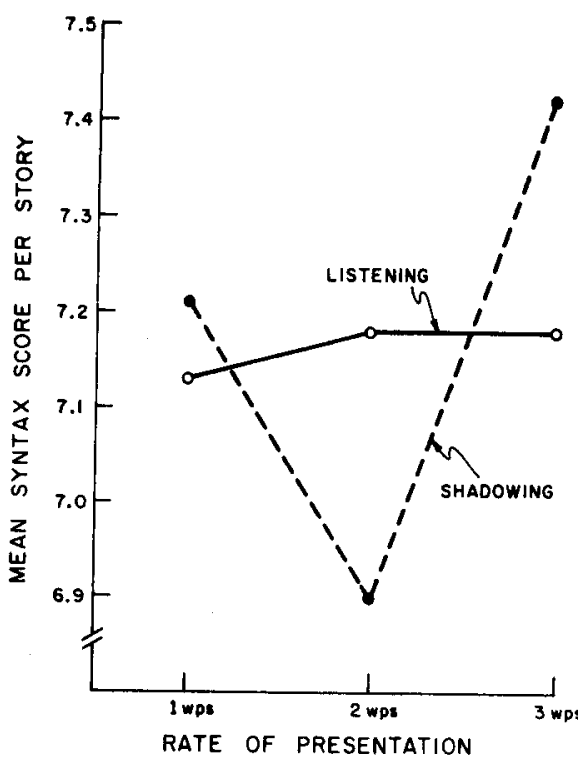

Fig. 3. Mean syntax recognition scores for listeners and shadowers.

shadowing facilitation hypothesis. For syntax, shadowers' scores were higher at 1 and 3 wps and lower at 2 wps, but none of these differences approached significance. The syntax comparisons neither support nor deny the hypothesis. In summary, the differences between listening and shadowing are not sufficiently pronounced for us to consider the shadowing facilitation hypothesis as confirmed, and another hypothesis for the effect of shadowing on retention will be considered below.

In addition to analyses of retention scores, analyses of variance on shadowers' speaking errors were carried out. Separate analyses showed that distortions, omissions, and substitutions became much more frequent as rate of presentation increased, and rate was a highly significant variable in all three cases $[F(2,60)>10.0$, $\mathrm{p}<.001$ in all cases]. In addition, as cerebral dominance theories would predict (Kimura, 1964), when a story was presented to a S's left ear, there was a tendency for more omissions and substitutions to occur than when the story was presented to his right ear. This tendency was significant for substitutions $[t(35)=1.87, \mathrm{p}<.05]$, but it did not reach significance for omissions. For percentages of distorted words the opposite trend was noted; more distortions occurred for presentation of a story to a S's right ear, but this difference was not significant. When the interaction of substitutions and distortions by ears was examined, however, the right-ear tendency for distortions was sufficient to produce a significant interaction $[t(70)=2.11$, $p<.05]$. All other terms of the analysis of variance of speaking errors were insignificant.

The range of shadowers' speaking errors was from 17 to 236 errors. In order to study differences between good and bad shadowers, the two best shadowers and two of the worst were selected for an analysis of their mean latencies. Table 1 shows that the mean latency of the poor shadowers was greater than that of the skillful shadowers at all rates. This main effect was not significant, but at 2 and 3 wps the difference was significant [2 wps: $t(6)=12.65, \quad p<.01 ; 3$ wps: $t(6)=5.20, p<.01]$. The mean latency of the poor shadowers increased as rate of presentation increased, whereas for the skillful shadowers latency decreased with increasing rate. The interaction of shadowing skill with rate, however, did not reach significance. There was no significant difference between latencies for delivery to the left ear vs the right ear.

\section{Retention Score Correlations}

Correlations of shadowers' retention scores with their other retention scores are displayed in Table 2. The table shows that correlations of content scores with syntax scores were significantly positive at all rates and that no other correlations approached significance. Thus, at each rate shadowers who made high content scores showed a strong tendency to obtain high syntax scores as well.

Correlations among listeners' retention scores are displayed in Table 3. Like the shadowers' scores, those of listeners showed a positive correlation between content and syntax at all rates, but this positive correlation was significant only at 2 wps. Unlike those of shadowers, however, listeners' retention scores contain other significant correlations. At 2 and 3 wps listeners' word scores were positively correlated with their content scores, and the correlation was significant. Also, at 2 wps listeners' word scores showed a significant positive correlation with syntax scores. At 2 wps, therefore, listeners' retention scores showed a significant positive correlation with every other retention score.

For each of the correlation coefficients on listeners' scores, $95 \%$ and $99 \%$ confidence limits were computed (Hays, 1963). The corresponding coefficients of the shadowing group were then examined. In three cases shadowers' coefficients were so different that they fell outside the listeners' confidence limits. At 2 wps shadowers' word-content and word-syntax correlations differed from listeners' correlations at the $1 \%$ level, two-tailed. At 1 wps the shadowers' word-syntax correlation differed from the listeners'
Table 1

Mean Latency of the Shadowing Response

\begin{tabular}{cccc} 
& $1 \mathrm{wps}$ & $2 \mathrm{wps}$ & $3 \mathrm{wps}$ \\
\hline $\begin{array}{c}\text { Good } \\
\begin{array}{c}\text { Shadowers } \\
\text { Poor }\end{array}\end{array}$ & $1.09 \mathrm{sec}$ & $0.77 \mathrm{sec}$ & $0.76 \mathrm{sec}$ \\
Shadowers & $1.25 \mathrm{sec}$ & $1.68 \mathrm{sec}$ & $2.40 \mathrm{sec}$ \\
\hline
\end{tabular}

Table 2

Correlations Among Shadowers' Retention Scores

\begin{tabular}{lccl}
\hline & 1 wps & 2 wps & 3 wps \\
\hline Words: content & 0.173 & -0.157 & 0.181 \\
Content: syntax & $0.514 \mathrm{a}$ & $0.413 \mathrm{~b}$ & $0.386 \mathrm{~b}$ \\
Words: syntax & -0.209 & -0.023 & 0.097 \\
\hline
\end{tabular}

Note-All t tests are two-tailed with $34 d f$

aSignificant at $p<.01$.

$b_{\text {Significant at } p<.05}$

Table 3

Correlations Among Listeners' Retention Scores

\begin{tabular}{lllr}
\hline & 1 wps & 2 wps & 3 wps \\
\hline Words: content & 0.125 & $0.475^{\mathrm{a}}$ & 0.477 \\
Content: syntax & 0.238 & $0.413^{\mathrm{b}}$ & 0.235 \\
Words: syntax & 0.165 & $0.471^{\mathrm{a}}$ & -0.152 \\
\hline
\end{tabular}

Note-All $t$ tests are two-tailed with 34 df. ${ }^{a}$ Significant at $p<.01$.

$b_{\text {Significant at } p<.05}$

correlation at the $5 \%$ level, two-tailed. These differences between shadowers and listeners suggest that shadowing markedly altered the listening strategy of shadowers.

\section{Retention Scores}

\section{DISCUSSION}

Aaronson (1967) has noted that most short-term memory experiments in which rate was varied suggest that faster rates, which reduce the time available for perception, produce lower accuracy. The present experiment on listening and shadowing provided data from word recognition and content retention to support the generalization. The lack of a regular effect of rate on syntax scores for either group-which is an exception to the rule--has possible explanations. First, since the syntax test contained the answers, in effect, for the other two tests, it had to be administered last. Considerable forgetting relative to the other tests could have occurred, and this forgetting may have obscured a real effect of rate. In support of this account for the absence of rate effect is the fact that remembering exact syntactic form is not a well-practiced skill and may be especially susceptible to forgetting. Sachs (1967) tested syntax recognition after 0,80 , or 160 intervening syllables and found that memory for syntactic changes after 80 or 160 syllables was near the chance level. Second, it is possible that there really is no monotonic effect of rate on syntax scores: Ss may use different listening strategies at the different 


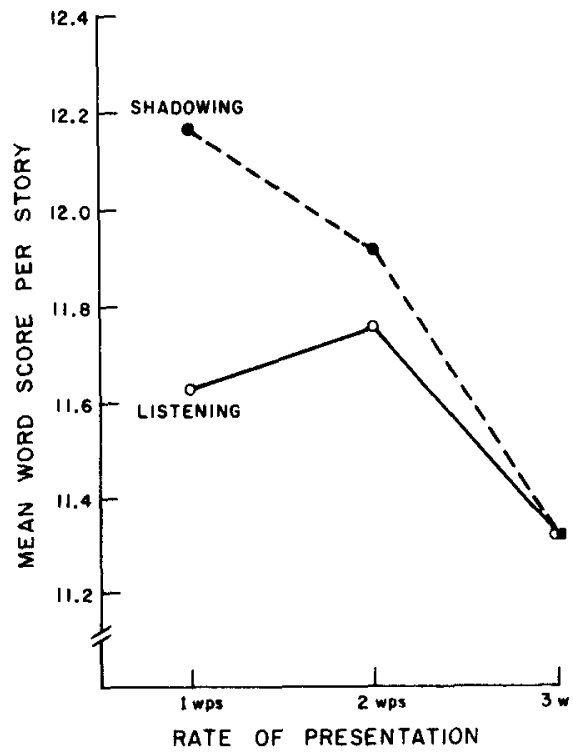

Fig. 1. Mean word recognition scores for listeners and shadowers.

Ss answered every item in all tests, guessing if necessary. For the word-recognition test they circled "yes" if the word printed on the sheet occurred in the story and "no" otherwise. For the content test they chose the correct alternative. For the syntax-recognition test they chose the phrase that contained the exact wording of the story. Ss were nun individually in sessions that averaged $80 \mathrm{~min}$ in duration. In the few minutes during which an $\mathrm{S}$ listened to a story, he wore Grason-Stadler Model D30 stereophonic earphones in an Industrial Acoustics soundproof booth. When taking the tests, he removed the earphones, and the door to the booth was opened.

The $36 \mathrm{Ss}$ in the shadowing group heard the practice stories in both ears and shadowed them. They spoke with normal effort into a small Voice of Music microphone that hung from the ceiling of the booth. After shadowing the practice stories, they proceeded to the six experimental stories, which they heard monaurally and shadowed. They repeated the words aloud in a normal loudness and were told to start repeating after a few words had been spoken-not to wait until a whole sentence had passed before beginning to shadow. The E monitored the S's shadowing response in earphones outside the booth, and, if during the practice session an S spoke too loudly, too softly, or too indistinctly, he was coached in correct technique. When an $S$ had finished the practice, he was informed that he would hear a series of stories and that he should try to make as few speaking errors as possible while trying also to understand the story. He was also informed that tests would be administered on the details of each story as soon as he had finished it. The arrangement of stories, ears, rates, and tests was identical to that of the listening group.

The Ss' shadowing responses were recorded on one track of a Sony Model TC-200 stereophonic tape recorder, and the message they were shadowing was re-recorded on the other track. The resulting stereophonic recording was subsequently used to determine the mean latency of the Ss' shadowing responses.

\section{SHADOWING ERRORS}

The recorded shadowing responses were analyzed according to four categories of errors: (1) omissions of words; (2) substitutions of one recognizable English word for a word in the story; (3) distortions of the phonemes or temporal patterns within a word, such as the omission, repetition, prolongation, or anomalous stressing of syllables; and (4) additions of words or transpositions of the order of words.

An analysis of variance according to Winer (1962, Chap. 8) was carried out on the total number of words omitted in the various conditions, and a separate analysis of variance was carried out on the total number of substituted words. The numbers of distorted words were not analyzed in their raw form but were transformed to percentages of the words spoken by the $S$ that were distorted. An analysis of variance was carried out on these percentages for the various conditions. No analysis of variance was carried out on the fourth category of error, since these errors accounted, for less than $5 \%$ of the total number of errors.

To determine the mean latency of the shadowing response for a subgroup of 4 of the 36 shadowers, 17 words were selected pseudorandomly from each story. Latencies were measured by stopwatch for each of the 17 words when possible, and the mean per story was obtained. Sometimes omissions of words reduced the number of available items below 17. In all cases the mean for available data was used for analysis.

\section{RESULTS}

Figures 1 and 2 show that word-recognition scores and content scores declined as the rate of presentation increased. For the combined shadowing and listening groups, the effect of rate was significant on both measures [words: $F(2,120)=6.08, \quad p<.01 ;$ content: $F(2,120)=8.97, \quad p<.01]$. Rate of presentation was also significant for the shadowing group alone [words:

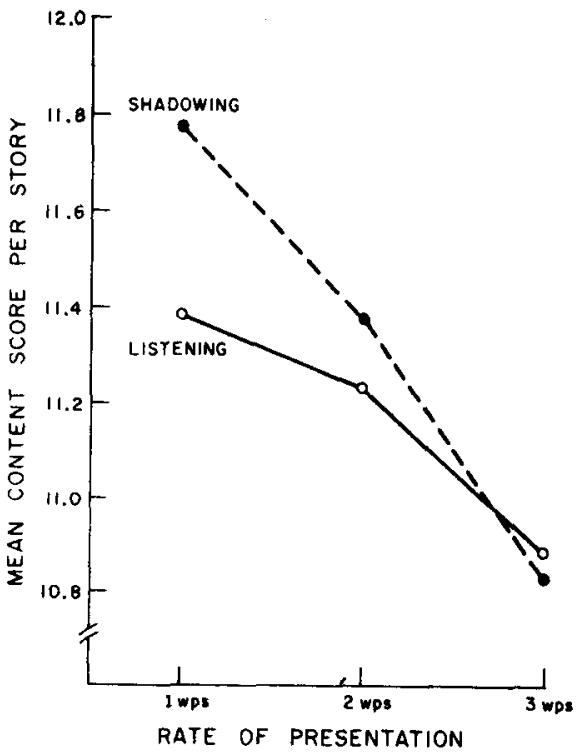

Fig. 2. Mean content scores for listeners and shadowers.

$F(2,60)=6.69, \quad p<.01 ;$ content: $F(2,60)=7.02, p<.01]$, but for listeners it did not reach significance. Statistical tests are one-tailed unless specified otherwise. Tests are two-tailed only when no a priori hypothesis could be adduced. Rate was not a significant component in the separate analysis of syntax scores for either group of Ss. Stories and ears were not significant sources of variance for either group on any of the three retention measures, and interactions were insignificant.

A closer examination of the retention scores in Figs. 1, 2, and 3 reveals that rate had a much greater effect on shadowers' scores than on listeners' scores. Across the three measures, the mean retention score change associated with a change in presentation rate was larger for shadowers than for listeners $[\mathrm{t}(10)=3.50, \mathrm{p}<.01]$.

The shadowing facilitation hypothesis predicted that successful shadowing should lead to higher retention scores than simple listening. The retention data show no significant overall difference between shadowing and listening, but at the individual rates there are some differences. At 1 wps shadowers' mean scores on word recognition and content retention were higher than listeners', but the difference did not quite reach significance [words: $t(70)=1.59, \quad p=.06 ;$ content: $t(70)=1.37, p=.09]$. At 2 wps the shadowers' scores on these two measures were higher than the listeners' but not significantly so. At 3 wps the difference disappeared for word recognition and was in the opposite direction for content. These comparisons tend to support the 
rates. It is likely that since syntactic units are long with respect to words and much semantic information, different rates can cause the $S$ to shift from one listening strategy to another, whereas a strategy for semantic information can remain the same in the face of changes in rate.

The observation that rate affected shadowers' retention scores more than listeners' scores is largely a result of the fact that shadowing a message at $3 \mathrm{wps}$ is much more difficult than shadowing a message at 2 wps. Every $S$ was willing to admit this fact, and speaking error scores also support it. By contrast, the increase in difficulty for listeners is small.

The shadowing-facilitation hypothesis, viz, that successful shadowing improves retention with respect to listening, was only very tentatively confirmed. The fact that the difference in content scores at 1 wps did not reach significance may perhaps be explained by the ceiling effect, since the mean score was close to 12 items correct in 14. If a more difficult test of semantic retention had been employed, the difference might have been larger. The fact that the difference on word recognition at 1 wps did not quite reach statistical significance may reflect the possibility that differences on this measure are truly small.

Although the experiment provided scant evidence that shadowing raises retention scores, the evidence from correlational analyses quite firmly establishes that shadowing alters $S s^{\prime}$ retention in some way. The primary effect of shadowing was to isolate success in word retention from the other forms of retention. As noted above, whereas listeners' word scores showed significant positive correlations with other retention scores in three instances, shadowers' word scores never showed a significant correlation with other scores. Furthermore, shadowers' correlations of word scores with other scores were significantly lower than listeners' correlations in three cases. In listening, then, an S's ability to retain one form of linguistic information is roughly equivalent to his ability to retain other forms of information. If he is good at word retention relative to other listeners, he tends to be good at content and syntax retention as well. In shadowing, an S's ability to retain syntax tends to be equivalent to his ability to retain semantic content, but his retention of words shows no relation to retention of the other two forms of information. Some aspect of the shadowing process must alter his retention of words.

That aspect of shadowing which affects word retention may be the monitoring of an S's own voice. When an $S$ listens to a message without shadowing, he is free to devote his auditory attention to the input message exclusively. When he shadows the message, however, two auditory signals compete for his attention: (1) the input message and (2) his own voice. Since it is reasonable to suppose that an $S$ occasionally monitors his own voice while shadowing, it follows that his auditory attention must switch back and forth between the input message and his own voice. The switching, in itself, is not sufficient to account for the effect of shadowing on word retention, since no claim concerning the periodicity of the switching process has yet been substantiated. Evidence from experiments in which delayed auditory feedback has been introduced into the shadowing process, however, allows some elaboration of the monitoring hypothesis. Carey (1968) found that the best way to explain the results of shadowing experiments involving delayed auditory feedback was to suggest that an $S$ normally monitors his own speech production on the level of individual syllables and on the level of short phrases a word or two in length. If this monitoring hypothesis is correct, it can help to explain the effect of shadowing on word retention. Monitoring of spoken output on the level of syllables and short phrases would tend to improve retention of words, while having little effect on retention of content and syntax, which are arranged in considerably longer units. At slow rates, when there is plenty of time for the processes of attending to the input message, organizing the shadowing response, and monitoring the spoken output, monitoring can help an $S$ retain words that he might not retain as a listener. His abilities in retaining syntax and semantics remain positively correlated as in listening. Shadowers' word scores, modified by monitoring, thereby show a near-zero correlation with their other retention scores. At faster rates, monitoring still has the effect of dissociating word retention from an $S$ 's abilities with syntax and semantics, but because of the time pressures of the shadowing task, no absolute improvement over listeners' word scores is observed.

The suggestion that successful shadowing employs self-monitoring, which improves word retention, may have general implications for experiments on selective attention. If subsequent experiments in which an $S$ shadows one of two dichotic stories yield similar results, then part of the explanation for the difficulty in recalling material in the unattended message may be that the mechanisms required in shadowing have preempted attentive capacity. In other words, if shadowing requires complex monitoring, which improves word retention, shadowing may be so difficult that attention to another linguistic channel is precluded. The selectivity of dichotic shadowing experiments may be not so much a phenomenon of filtering or suppression as a simple incapacity resulting from the demands of the shadowing task itself. This line of reasoning may account for Treisman's observation that "Ss find shadowing helps them ignore the competing unattended message."

\section{Speaking Errors}

The fact that shadowers who made few errors were closer temporally to the input message than the worst shadowers suggests that errors may occur partly as a result of a failure of auditory short-term memory. Neisser (1967) has reviewed considerable evidence that the normal duration of echoic memory is about $1 \mathrm{sec}$. It seems likely, therefore, that when shadowing latencies are appreciably longer than $1 \mathrm{sec}$, as they were for the poor shadowers at the faster rates, some semantic and syntactic aspects of the input message will be lost. At these latencies, therefore, considerable forgetting can occur, and omissions and substitutions can appear in the shadowing response. It is unclear whether the poor shadowers simply adopted an ineffective shadowing strategy or whether their poor performance is a reflection of basic individual differences in linguistic performance.

Numerous studies (e.g., Kimura, 1961, 1964; Broadbent \& Gregory, 1964) have observed right-ear superiority for verbal material presented dichotically. In these studies, which typically use digits, the asymmetry is observed in recall or in recognition of the material presented to the separate ears. The current observation, to the effect that substitution errors are less frequent when input is to the right ear, may be the first report of an asymmetry in speech production in normal Ss. The explanation for the asymmetric pattern of substitutions may well be in terms of asymmetric perception of the input message, in which case the current observation becomes an extension of Kimura's findings. If the explanation for the asymmetry is in terms of perception, one would expect the current study to contain asymmetries in retention scores as well, but no such asymmetries were detected. This discrepancy may be explained by the possibility that real retention asymmetries may have been present but that the retention tests, which by no means covered all the information in the stories, were not sensitive enough to detect them. If more sensitive tests of retention after shadowing are developed, it will be interesting to examine in detail the 
relation between shadowing errors and retention errors. In particular, a question to be considered is whether some linguistic levels, such as syntax, are more highly lateralized than other levels.

\section{REFERENCES}

AARONSON, D. Temporal factors in perception and short-term memory. Psychological Bulletin, 1967, 67, 130-144.

BROADBENT, D., \& GREGORY, M. Accuracy of recognition for speech presented to the right and left ears. Quarterly Journal of Experimental Psychology, 1964, 16, 359-360.
CAREY, P. Delayed auditory feedback and the shadowing response. Unpublished doctoral dissertation, Harvard University, 1968.

CHERRY, C. Some experiments on the recognition of speech with one and with two ears. Journal of the Acoustical Society of America, 1953, 25, 975-979.

HAYS, W. L. Statistics for psychologists. New York: Holt, Rinchart, \& Winston, 1963.

KIMURA, D. Cerebral dominance and the perception of verbal stimuli. Canadian Journal of Psychology, 1961, 15, 166-171.

KIMURA, D. Left-right differences in the perception of melodies. Quarterly Journal of Experimental Psychology, 1964, 16, 355-358.
MORAY, N. \& TAYLOR, A. The effect of redundancy in shadowing one of two dichotic messages. Language \& Speech, 1958, 1, 102-109.

NEISSER, U. Cognitive psychology. New York: Appleton-Century-Crofts, 1967.

SACHS, J. Recognition memory for syntactic and semantic aspects of connected discourse. Perception and Psychophysics, 1967, 2, 437-442.

WINER, B. Statistical principles in experimental design. New York: MeGraw-Hill, 1962.

(Accepted for publication May 15, 1970) 